\title{
A Smart Drug Delivery Silica Nanoparticles System for Diabetes Mellitus Disease Treatment
}

\author{
Dalia A Elaty ${ }^{1}$ and Mark George Fayez Fakhry ${ }^{2}$ \\ ${ }^{1}$ Lecturer of Pharmaceutics, Faculty of Pharmacy, October University for Modern Science and Arts, MSA University, Egypt \\ ${ }^{2}$ Student, Faculty of Pharmacy, October University for Modern Science and Arts, MSA University \\ *Corresponding Author: Dalia A Elaty, Lecturer of Pharmaceutics, Faculty of Pharmacy, October University for Modern Science and Arts, \\ MSA University, Egypt.
}

Received: November 20, 2019; Published: November 26, 2019

DOI: $10.31080 /$ ASPS.2019.03.0444

\begin{abstract}
Type 2 diabetes makes up approximately $85 \%$ of all diabetic cases and it is linked to approximately one-third of all hospitalizations. Diabetes mellitus is a metabolic disorder that can lead to complications such as kidney failure, blindness, ketoacidosis, stroke and heart diseases. Types of diabetes mellitus are Type 1 diabetes mellitus, Type 2 diabetes mellitus and Gestational diabetes mellitus. Drugs used in the treatment of diabetes are insulin, sulphonylureas, biguanides, thiazolidinediones, $\alpha$-glcosidase inhibitors and dipeptidyl peptidase IV inhibitors. Newer therapies with long-acting biologics such as glucagon-like peptide-1 (GLP-1) analogues have been promising in managing the disease, but they cannot reverse the pathology of the disease. Additionally, their parenteral administration is often associated with high healthcare costs and risk of infections. .Oral delivery of these compounds would significantly improve patient compliance; however, poor enzymatic stability and low permeability across the gastrointestinal tract makes this task challenging. Nanotechnology is the use of nanomaterial's which are smaller than $100 \mathrm{~nm}$ either be natural or manmade. Silica nanoparticles possess numerous advantages including optimum biocompatibility and bio distribution. Silica nanoparticles (SNPs) are emerging functional inorganic nanomaterials which offer an alternative to traditional carriers for oral delivery of peptides and drugs ; Modification of SNPs with appropriate functional groups improves control over the loading/release of payload for oral delivery. Metformin was loaded by SiO2 nanoparticles to release the drug gradually, glimepiride was loaded on both MCM-41 and Hollow mesoporous silica nanoparticles for the improvement of solubility and insulin was loaded on the mesoporous silica nano particles to control its release. MSNs like MCM-41, MCM-48, SBA-1, SBA-3, SBA-15, SBA-16 are tested to drugs delivery for different diseases e.g. inflammation, cancer, diabetes, neurological disorders and even as a carriers in gene therapy or biosensor in diagnosis. The reasons of their popularity are that these materials have a several advantages, such as unique ordered structure of high specific area and pore volume and consequently high sorption capacity. Moreover production of these materials is relatively simple, inexpensive and easy controllable. Finally, they are nontoxic and biocompatible which are the key requirements.
\end{abstract}

Keywords: Diabetes Mellitus; $\beta$ Cells; $\mathrm{SiO}_{2}$

\section{Diabetes mellitus}

Szablewski [1] cited that diabetes mellitus is a metabolic disorder that lasts for the lifetime of a person and can lead to complications such as kidney failure, blindness, ketoacidosis, stroke and heart disease. Symptoms of diabetes mellitus include polydipsia, polyuria, polyphagia, blurred vision, fatigue and poor wound healing. Type 1 diabetes mellitus is an organ specific autoimmune disease that is attributed to the specific adaptive immunity against the antigens of the $\beta$ cells. Despite the fact that type 1 diabetes mellitus occur at any age, it is diagnosed in most of the cases before the age 
of 30. Rodriguez-Sosa., et al. [2] stated that type 2 diabetes mellitus happens due to the altered response to the insulin and the pancreatic $\beta$ cells failure to secrete the required amount of insulin in order to optimize the blood glucose levels. Moreover, type 2 diabetes mellitus is accompanied by increase in the serum levels of inflammatory cytokines including TNF- $\alpha$, IL-12 and MIF [2]. Type 2 diabetes mellitus is usually accompanied by hypertension, hypercholesterolemia, hypertriglyceridemia and obesity [3]. Gestational diabetes mellitus is a condition in which there is an elevated glucose levels during pregnancy. Arafa and Dong [4] mentioned that women with a history of depression are at increased risk for gestational diabetes. Saeedi., et al. [5] stated that 463 million people have diabetes in 2019 mentioning that about a billion people are living with diabetes. In addition, it is predicted that in 2030 578 million people will have diabetes and this number is subject to increase by $51 \%$ in 2045 .

\section{Conventional antidiabetic drugs in market}

Howland and Mycek [6] stated that anti diabetic drugs include insulin, insulin secretagogues such as first and second generation of sulphonylureas, biguanides, thiazolidinediones, $\alpha$-glcosidase inhibitors and dipeptidyl peptidase IV inhibitors. Insulin works by stimulating the uptake of glucose, increase glycogen synthesis in liver, increased protein synthesis and increasing the triglyceride synthesis and storage by the adipocytes. Sulphonylureas which include tolbutamide and glimpiride works by enhancing the insulin secretion from the $\beta$ cells in pancreas and also by the reduction of hepatic glucose output [6]. Biguanides include metformin which works by inhibiting of the hepatic glucose output and promoting the uptake of glucose by fat and muscles. Thiazolidinediones include pioglitazone and rosiglitazone which works by stimulating peroxisome proliferator activated receptor gamma which in turn promote the glucose uptake by fat and muscles. A-glucosidase inhibitors which include acarbose inhibit the $\alpha$-glucosidases which results in slower absorption of carbohydrates. dipeptidyl peptidase IV inhibitors which includes saxagliptin inhibit the breakdown of GLP-1 and thus allow increased insulin secretion and decreased hepatic glucose production.

\section{Nanotechnology applications in biomedical field}

Chen., et al. [7] cited that nanomaterials are those particles which are smaller than $1000 \mathrm{~nm}$. These materials possess different physical properties from the bulk materials. Nano particles used in biology or medicine are either natural or manmade. The manmade nanoparticles include grapheme, carbon nanotubes, liposomes, dendrimers, silicon, super paramagnetic iron oxide and silica nanoparticles. The natural nanoparticles used in the field of nanomedicine are the DNA. Nanoparticles possess large surface area and high surface to volume ratio. They can be compared to the size of antibodies enzymes and DNA as well as the cell products such as exosomes. The large surface area results in a high loading capacity of the molecules. The factors which facilitate the biodistribution of the nano particles are size, dispersity, surface charge and hydrophobicity. The particles which are smaller than $8 \mathrm{~nm}$ are eliminated by the kidneys rapidly while those between 30 and 200 nm have shown the ability to accumulate in tumours via the enhancement permeability and retention effect [7]. Surface properties play a major role in the nano particles as any alteration in the chemistry of the surface of the particle will lead to a change in the size and charge and thus its reactivity. Nanoparticles differ from each other in the endogenous properties. Carbon nanotubes can penetrate through different barriers in the cell and the void area present in center can be used for delivery of drugs. Graphene has a high surface area which in turn gives it high adsorption characteristics. Liposomes which have a lipid bilayer that is similar to the biological membranes have been used to load therapeutic molecules, fluorocarbon gas and nucleic acids in order to deliver the drugs. Superparamagnetic iron oxide nano particles are used as MRI contrast agents. Nanomedicine which depends on the nanomaterials has been used to treat numerous diseases such as cancer, heart diseases and diabetes.

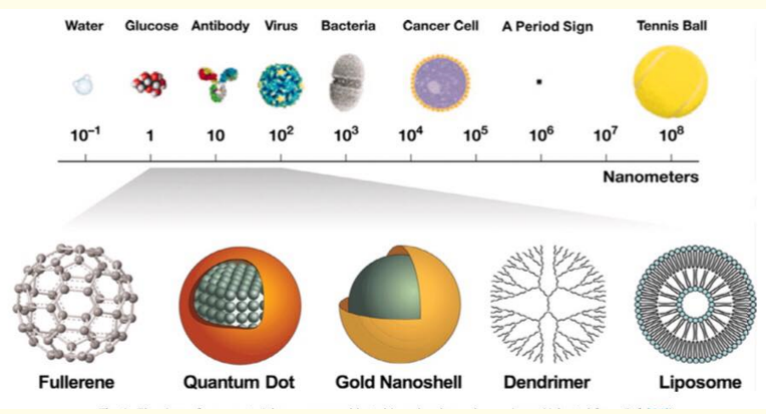

Figure 1: The size of nanomaterials are comparable to biomolecules and organisms. 


\section{Silica nanoparticles applications in medical fields}

Silica nanoparticles have numerous advantages for to be used in medicine. The easy modifying of the particles makes it biocompatible and gives it the optimal biodistribution. Modifications can be spacers for colloidal stability, antibodies for active targeting, gating molecules for drug release stimulation, fluorophores for imaging [8]. The good biocompatibility of silica nanoparticles arises from their degradation to silicic acid which is nontoxic [7]. In addition, the decrease in the hydroxyl groups in the surface of the nano particles increases the biocompatibility. Silica nano particles carry the drug in their mesopores and release the drug via diffusion. Mesoporous silica nanoparticles are one of the most important forms of silica nano particles. All mesoporous silica nano particles have 3 major components which are solid support, payload for the delivery of drugs and external enginery [9]. The diameter of the mesoporous silica nanoparticles ranges from 2 to $50 \mathrm{~nm}$.

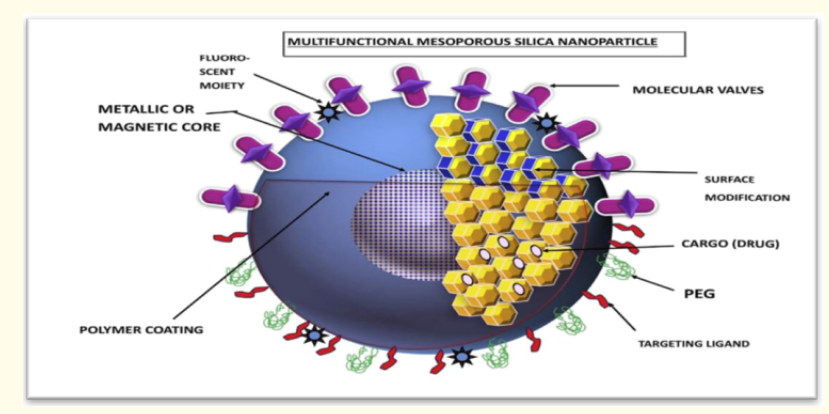

Figure 2: Showing the structure of multifunctional mesoporous silica nano particles.

\section{Loading of antidiabetic drugs in silica nano particles}

Patiño-Herrera., et al. [10] formulated metformin by $\mathrm{SiO} 2$ nanoparticles pellets for the gradual release of the drug. Metformin $\mathrm{Hcl}$ is one of the most widely used drugs for the treatment of diabetes mellitus. the major problem that is shown when a drug is ingested via the oral route is drug dissolves rapidly in the stomach and does not produce the adequate levels in the blood and when the adequate level is reached it decreases and becomes necessary to ingest another dose in a few hours. This can be solved by adsorbing the drug in mesoporous silica nanoparticles and further compression to be in the form of pellets. The MSNPs does not swell in solvents, has large surface area, appropriate pore size and thermal insulation. Tzankov., et al. [11] loaded the poorly soluble glimpiride on the hollow mesoporous silica nanoparticles in order to improve its solubility.

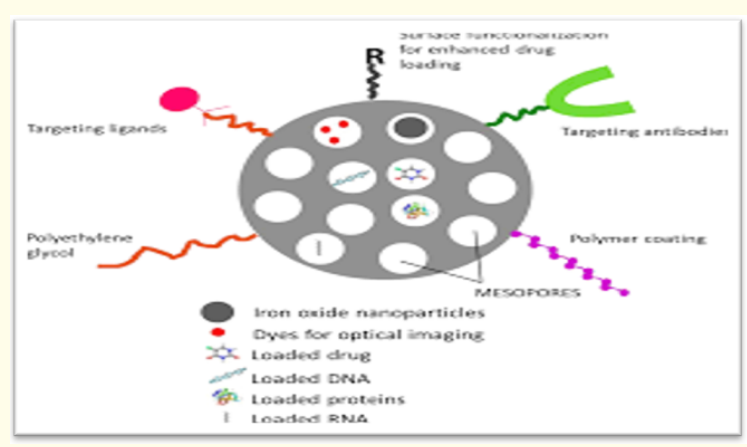

Figure3

MCM-41 (MCM-41 is a mesoporous material with a hierarchical structure from a family of silicate and alum silicate solids that were first developed by researchers at Mobil Oil Corporation and that can be used as catalysts or catalyst supports) is a hexagonal pore structure and matrix confirmation that serves as a drug carrier. The MCM-41 pore size ranges from 2-5 $\mathrm{nm}$. In addition, Hollow mesoporous silica has a higher storage capacity due to the hollow core structure. Elsayed., et al. [12] used mesoporous silica nano particles as insulin carriers because of their biocompatibility and high pore volume which controls the release of insulin. The insulin mesoporous silica nano particles were then capsulated by chitosan which acts as a gatekeeper. Chitosan was chosen due to the high potential in controlling its degradation rate and in turn affects the insulin release rate.

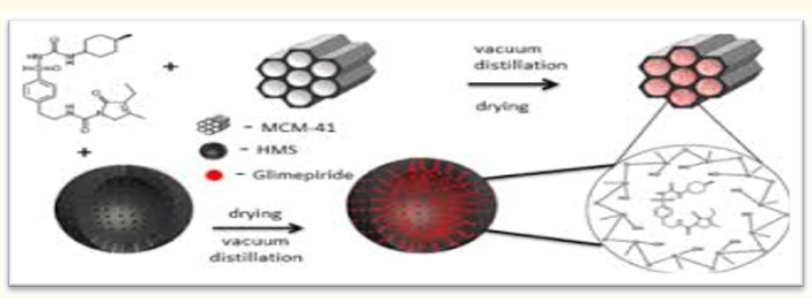

Figure 4: Poorly soluble glimpiride drug. 


\section{Conclusion}

Mesoporous silica materials of different types are attractive from the point of view of drug loading in drug delivery systems. High pore volume and specific surface area are the important factors in drug loading process. Pore volume can't be totally filled with drug molecules in impregnation process and loading strongly depends on the interactions of drug with the mesoporous host and the rate of wettability by solution containing the drug. The wettability and the penetration of pores by wetting liquid are markedly improved in outgassed sample. The amount of drug deposited in pores depends on equilibrium of adsorption process. In the case of highly porous solids containing pores of small dimensions very important is the relation of pore dimension to dimensions of adsorbed molecules.

\section{Bibliography}

1. Szablewski L. "Role of immune system in type 1 diabetes mellitus pathogenesis". International Immunopharmacology 22 (2014): 182-191.

2. Rodriguez-Sosa M., et al. "Proinflammatory cytokine MIF plays a role in the pathogenesis of type-2 diabetes mellitus but does not affect hepatic mitochondrial function". Cytokine 99 (2017): 214-224.

3. Davis S. "Postprandial physiology and the pathogenesis of type 2 diabetes mellitus". Insulin 3 (2008): 132-140.

4. Arafa A and Dong J. "Depression and risk of gestational diabetes: A meta-analysis of cohort studies". Diabetes Research and Clinical Practice 156 (2019): 107826.

5. Saeedi P., et al. "Global and regional diabetes prevalence estimates for 2019 and projections for 2030 and 2045: Results from the International Diabetes Federation Diabetes Atlas, 9th edition". Diabetes Research and Clinical Practice (2019): 107843.

6. Howland R and Mycek M. Pharmacology. Philadelphia: Lippincott Williams and Wilkins (2006).

7. Chen F., et al. "Multifunctional nanomedicine with silica: Role of silica in nanoparticles for theranostic, imaging, and drug monitoring". Journal of Colloid and Interface Science 521 (2018): 261-279.
8. Mamaeva V., et al. "Mesoporous silica nanoparticles in medicine-Recent advances". Advanced Drug Delivery Reviews 65 (2003): 689-702.

9. Saroj S and Rajput S. "Composite smart mesoporous silica nanoparticles as promising therapeutic and diagnostic candidates: Recent trends and applications". Journal of Drug Delivery Science and Technology 44 (2018): 349-365.

10. Patiño-Herrera R., et al. "Prolonged release of metformin by SiO2 nanoparticles pellets for type II diabetes control". European Journal of Pharmaceutical Sciences 131 (2019): 1-8.

11. Tzankov B., et al. "Improvement of dissolution of poorly soluble glimepiride by loading on two types of mesoporous silica carriers". Journal of Solid State Chemistry 271 (2019): 253-259.

12. Elsayed A., et al. "Development of insulin loaded mesoporous silica injectable particles layered by chitosan as a controlled release delivery system". International Journal of Pharmaceutics 461 (2014): 448-458.

\section{Volume 3 Issue 12 December 2019}

(C) All rights are reserved by Dalia A Elaty and Mark George Fayez Fakhry. 